\title{
Altered Gut Microbiome under Antiretroviral Therapy: Impact of Efavirenz and Zidovudine
}

\author{
Shilpa Ray,* Aswathy Narayanan, Christian G. Giske, Ujjwal Neogi, Anders Sönnerborg, \\ and Piotr Nowak
}

Cite This: ACS Infect. Dis. 2021, 7, 1104-1115

Read Online

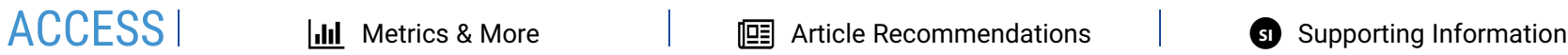

ABSTRACT: Millions of individuals currently living with HIV globally are receiving antiretroviral therapy (ART) that suppresses viral replication and improves host immune responses. The involvement of gut microbiome during HIV infection has been studied, exposing correlation with immune status and inflammation. However, the direct effect of ART on gut commensals of HIV-infected individuals has been mostly overlooked in microbiome studies. We used 16S rRNA sequencing (Illumina MiSeq) for determining the microbiota composition of stool samples from 16 viremic patients before and one year after ART. We also tested the direct effect of 15 antiretrovirals against four gut microbes, namely, Escherichia coli, Enterococcus faecalis, Bacteroides, and Prevotella to assess their in vitro antibacterial effect. 16S rRNA analysis of fecal samples showed that effective ART for one year does not restore the microbiome diversity in HIV-infected patients. A significant reduction in $\alpha$-diversity was observed in patients under non-nucleoside reverse transcriptase inhibitors; (NNRTI; 2 NRTI+NNRTI; NRTIs are nucleoside reverse transcriptase

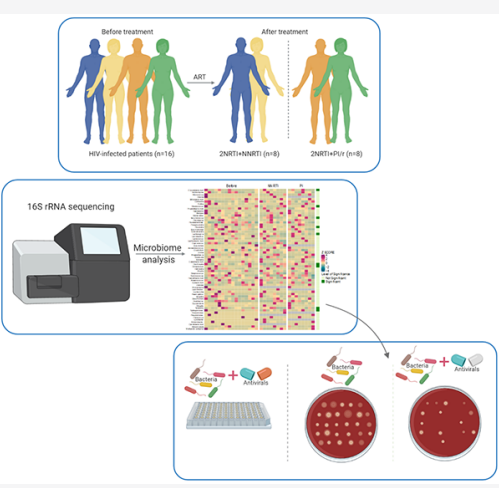
inhibitors) as compared to ritonavir-boosted protease inhibitors (PI/r; 2 NRTI+PI/r). Prevotella $(P=0.00001)$ showed a significantly decreased abundance in patients after ART $(n=16)$. We also found the direct effect of antivirals on gut microbes, where zidovudine (ZDV) and efavirenz (EFV) showed in vitro antimicrobial activity against Bacteroides fragilis and Prevotella. EFV also inhibited the growth of E. faecalis. Therefore, we observed that ART does not reverse the HIVinduced gut microbiome dysbiosis and might aggravate those microbiota alterations due to the antibacterial effect of certain antiretrovirals (like EFV, ZDV). Our results imply that restructuring the microbiota could be a potential therapeutic target in HIV-1 patients under ART.

KEYWORDS: gut microbiome, HIV, antiretroviral therapy, antibacterial effect, efavirenz, zidovudine

$\mathrm{T}$ he human gut comprises complex and diverse microbial communities that contribute to host immune responses, as well as cellular metabolism and nutrients acquisition. ${ }^{1}$ The gut microbiome also actively interacts with therapeutic drugs, making it an interesting target for modifying drug effectivity in individuals. This has been shown in several reports, where the therapeutic effect of drugs and vaccines was linked to the microbiome composition. ${ }^{2-6}$

More specifically, in recent years, the gut microbiome involvement in the pathogenesis of human immunodeficiency virus (HIV) infection is being increasingly recognized. ${ }^{7-9}$ The gut-associated lymphoid tissues (GALT) and epithelial cells, in fact, are majorly affected during HIV-1 infection, ${ }^{10}$ causing the GALT to experience loss of mucosal $\mathrm{CD}^{+} \mathrm{T}$-cells, which alters gut mucosal integrity, thereby disturbing gut homeostasis. ${ }^{10}$ Several studies have shown the alteration of the gut microbiome in HIV-infected individuals, with microbiome diversity correlating with immune status and inflammation. ${ }^{7,8,11,12}$ HIV-infected patients, in fact, have a less diverse microbial population as compared to uninfected individuals, with a correlation between $\alpha$-diversity and $\mathrm{CD}^{+} \mathrm{T}$ cell counts in untreated viremic individuals. The major genera affected during HIV infection are Bifidobacterium, Lactobacillus, Faecalibacterium, and Lachnospira. ${ }^{13,14}$ Additionally, different cohort studies have repeatedly found enriched Prevotella and Enterobacteriaceae at the genus and family levels, which can contribute to the inflammatory state in HIV infection. ${ }^{15-19}$

Antiretroviral therapy (ART) has changed the fate of HIV infection from lethal to chronic disease and leads to the control of HIV replication and recovery of immune function in most of the patients. However, the chronic inflammation and mucosal injury in the gastrointestinal tract are still present even during effective ART. Interestingly, the microbiome changes observed in untreated HIV subjects have also been found in patients treated for several years with effective anti-HIV therapy both in

Special Issue: Gut Pathogens

Received: July 27, 2020

Published: December 21, 2020 

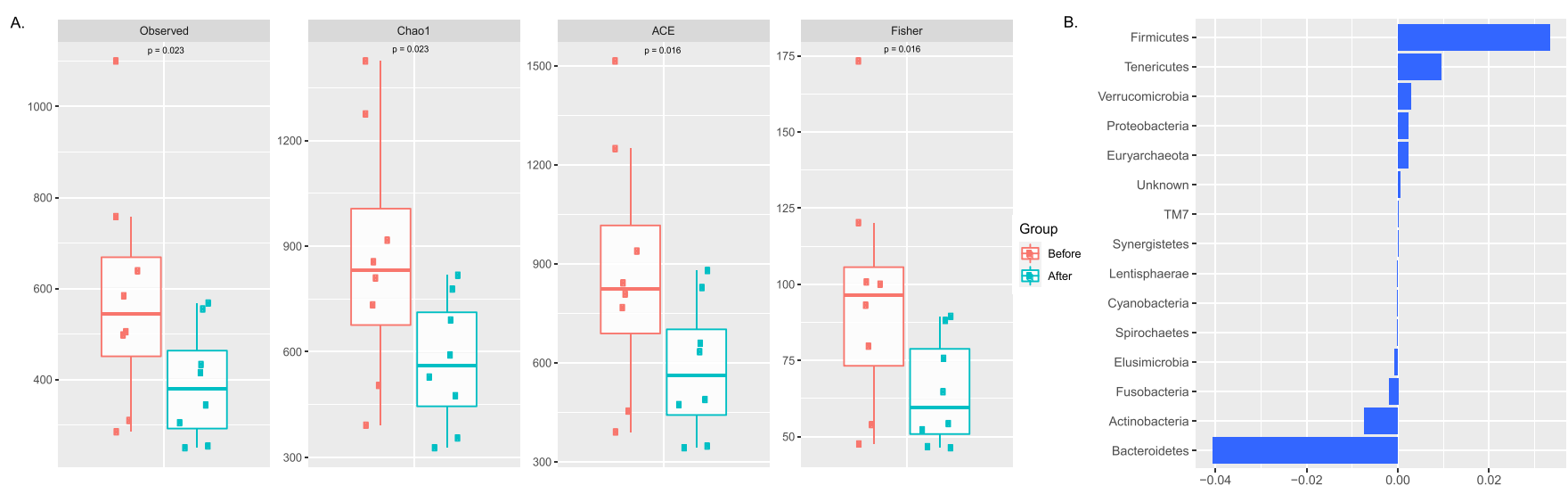

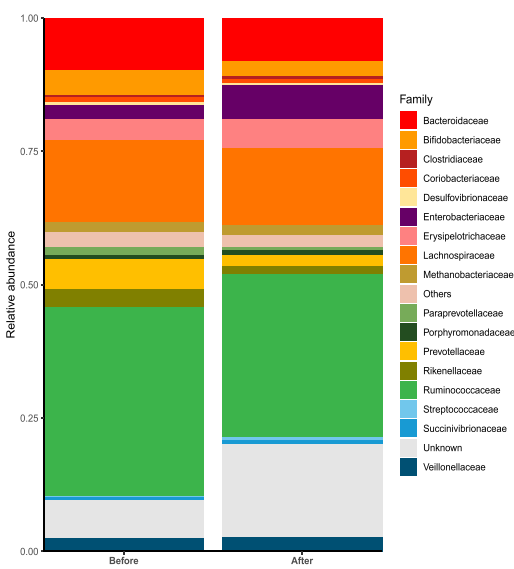

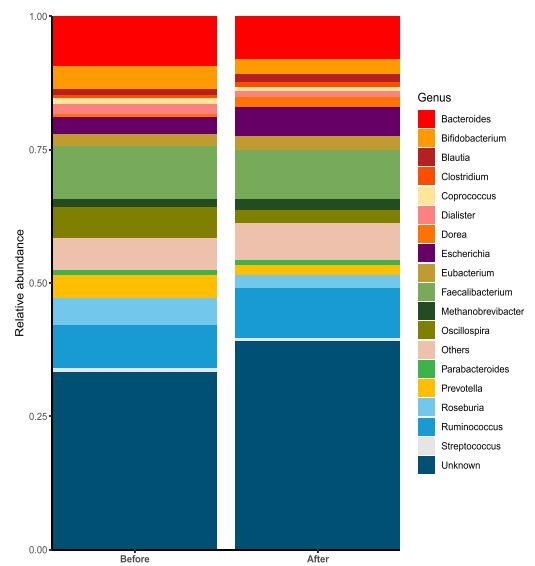

Figure 1. Changes in microbiota composition in individuals after NNRTI-based therapy (2 NRTI+NNRTI): (A) $\alpha$-diversity scores of the gut microbiota for evaluating the richness (Chaol, ACE, observed species) and diversity (Fisher) of bacterial communities in individuals before $(n=8)$ and after $(n=8)$ NNRTI-based therapy ( 2 NRTI+NNRTI) by Wilcoxon signed-rank test. We observed a significant reduction in $\alpha$-diversity in patients under NNRTI-based therapy relative to that before treatment. (B) PERMANOVA analysis of gut microbes at the phylum level in individuals under NNRTI-based treatment. (C and D) Bar plots depicting the relative abundance at the microbial family and genus levels in patients who received non-nucleotide reverse transcriptase inhibitors (2 NRTI+NNRTIs, $n=8)$.

cross-sectional and longitudinal studies. ${ }^{20-22}$ These observations have led to the hypothesis that antiretroviral drugs per se could aggravate the dysbiosis, which hinders the restoration of gut integrity in well-treated HIV-infected patients. ${ }^{8,23-25}$

In this work, we therefore aimed to explore if and which are the specific bacterial signatures in the gut microbiome before and after ART, as well as to evaluate the antibacterial effect of antiretroviral drugs in in vitro cultures.

\section{RESULTS}

Study Design. The study cohort consisted of 16 HIV-1infected patients who were viremic at baseline (median plasma HIV-1 RNA 52600 copies/mL, range 280-336000) with moderate immune deficiency as estimated by CD4 counts (median CD4+ T cell count: 360 cells $/ \mathrm{mm}^{3}$; range $\left.250-460\right){ }^{8}$ The patients received effective ART and were followed for a median period of 10 months. ART was composed of two nucleoside reverse transcriptase inhibitors (NRTIs) combined with either a non-nucleoside reverse transcriptase inhibitor (NNRTIs), $n=8$, or a protease inhibitor (PI), $n=8$ administered daily.

Changes in Bacterial Diversity of HIV-1 Patients after Antiretroviral Therapy (ART). Four $\alpha$-diversity indices were used to evaluate the richness (Chaol, ACE) and diversity (Fisher, Shannon index) of bacterial communities in patients after antiretroviral therapy $(n=16)$ compared to those before treatment (Figure S1A). A decreasing trend in the microbial diversity and richness was observed in patients after ART (Figure S1A, Fisher $P=0.14$, Chao $1 P=0.16$, ACE $P=0.16$, Shannon $P=0.16)$. Additionally, we also tested the effect of different treatment regimens (2 NRTI+NNRTI and 2 NRTI $+\mathrm{PI} / \mathrm{r})$ on $\alpha$-diversity, which is discussed in detail later. Moreover, $\beta$-diversity comparison (NMDS2 and NMDS1) revealed moderate differences in the clustering of microbial communities before and after ART (Figure S1B).

At follow-up (one-year effective ART), certain changes in the microbiota composition were observed at the phylum, family, and genus levels (Figure S2). Firmicutes, Proteobacteria, Bacteroidetes, and Actinobacteria were the most predominant phyla, accounting for $97 \%$ of microbiome (Figure S2A). At the family level, Prevotellaceae $\left(\log _{2}\right.$ fold change $-1.55, P=0.002$ ) showed significantly less abundance after ART relative to that before ART (Figure S2B,D). A total of 139 genera were identified. The genera of Lachnospira ( $\log _{2}$ fold change $-2.54, P$ $=0.0007)$, Butyricicoccus ( $\log _{2}$ fold change $\left.-1.11, P=0.006\right)$, Oscillospira ( $\log _{2}$ fold change $-1.1, P=0.03$ ), and Prevotella ( $\log _{2}$ fold change $-1.63, P=0.00001$ ) showed a significant depletion in their respective abundances in the fecal samples of patients after ART introduction (Figure S2C,D). Previously, several studies have linked the Prevotella population with HIVinduced inflammation, ${ }^{7,16,26-28}$ while others have associated Prevotella-rich microbiota with sexual preferences. ${ }^{24}$ 

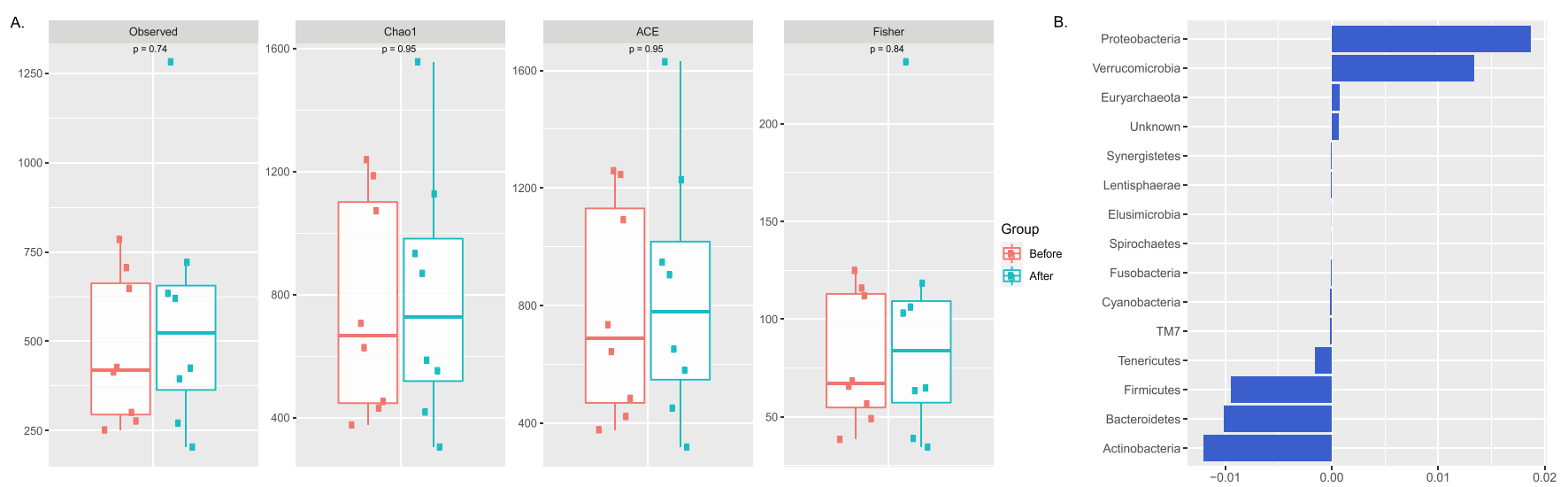

C.

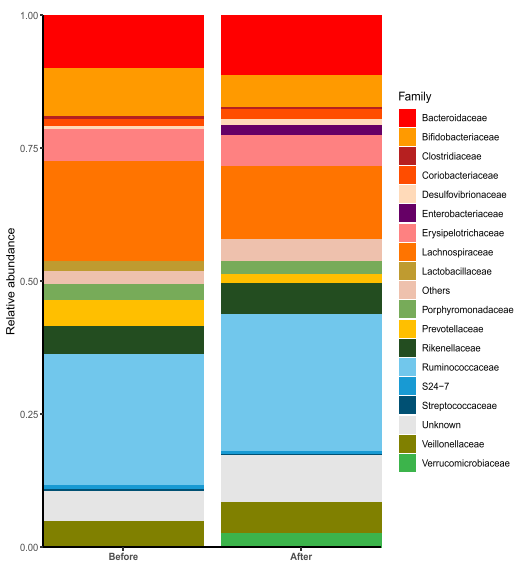

D.

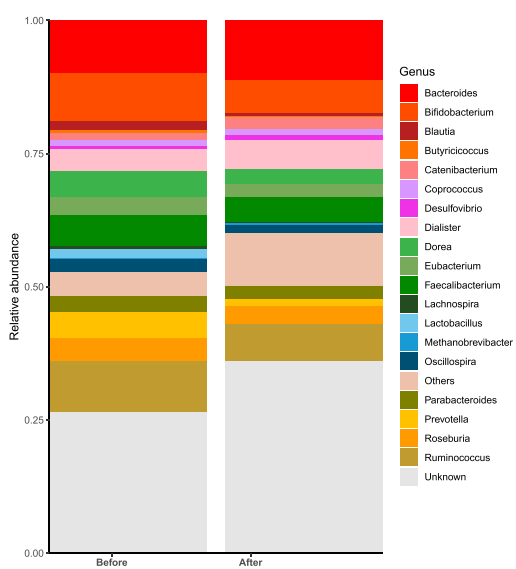

Figure 2. Changes in microbiota composition in individuals after PI-based therapy (2 NRTI+PI/r): (A) $\alpha$-diversity scores of the gut microbiota for evaluating the richness (Chao1, ACE, observed species) and diversity (Fisher) of bacterial communities in individuals before $(n=8)$ and after $(n=8)$ PI-based therapy (2 NRTI+PI/r) by Wilcoxon signed-rank test. (B) PERMANOVA analysis of gut microbes at the phylum level in individuals under PI-based treatment (2 NRTI+PI/r) (C and D) Bar plots depicting the relative abundance at the microbial family and genus levels in patients who received protease inhibitors $(2 \mathrm{NRTI}+\mathrm{PI} / \mathrm{r}, n=8)$.

Figure S3A depicts the taxonomic composition of microbial communities in the fecal samples of patients, defined as depleted or enriched in viremic subjects after antiretroviral therapy $(n=$ 16) as compared to before treatment. Similar observations were obtained as before, where Prevotella, Oscillospira, Bifidobacterium ( $\log _{2}$ fold change $-0.58, P=0.3$ ), and Bacteroides ( $\log _{2}$ fold change $-0.003, P=0.7)$ showed depletion after ART and Escherichia ( $\log _{2}$ fold change $1.15, P=0.2$ ) showed enrichment after ART. The size of the bubble was based on the Permutational multivariate analysis of variance (PERMANOVA) results (Figure S3B). The top bacterial genera, which were depleted and enriched after antiretroviral therapy, were obtained from PERMANOVA analysis, as represented in Figure S3B.

Differences in Gut Microbiota Composition of HIVInfected Individuals after NNRTI and PI-based Treatment. We noted significantly reduced $\alpha$-diversity indices in patients under NNRTI-based therapy (2 NRTI+NNRTI) (Figure 1A, Fisher $\mathrm{P}=0.016$, Chao $1 P=0.023$, ACE $P=$ 0.016 , observed species $P=0.023$ ) as compared to the individuals under PI-based therapy (2 NRTI+PI/r) (Figure 2A, Fisher index $P=0.84$, Chaol $P=0.94$, ACE $P=0.94$, observed species $P=0.74$ ), indicating a possible effect of NNRTI-based ART on gut richness and diversity. After NNRTI-based therapy, the proportion of Firmicutes and Bacteriodetes showed significant enrichment and depletion in abundance, respectively (Figure 1B). Prevotellaceae $\left(\log _{2}\right.$ fold change $-1.22, P=0.016)$ displayed a significant reduction in relative abundance after NNRTI-based treatment (2 NRTI + NNRTI) (Figure 1C and Figure S4A). However, at the genus level, Lachnospira ( $\log _{2}$ fold change $-2.15, P=0.007$ ), Oribacterium ( $\log _{2}$ fold change $-4.08, P=0.02$ ), Oscillospira ( $\log _{2}$ fold change $-1.31, P=0.05$ ), and Prevotella ( $\log _{2}$ fold change $-1.31, P=0.016)$ represent a significantly decreased abundance in patient fecal microbiota (Figure $1 \mathrm{D}$ and Figure S4A) treated with NNRTI regimen (2 NRTI+NNRTI).

After PI-based therapy, Proteobacteria showed a higher relative abundance whereas Firmicutes and Bacteriodetes were enriched in patients before PI therapy (Figure 2B). A significant decrease in the abundance of Clostridiaceae ( $\log _{2}$ fold change $-1.23, P=0.05)$ and Lactobacillaceae ( $\log _{2}$ fold change $-5.47, P$ $=0.0078)$ was observed in HIV-infected patients using NRTI $+\mathrm{PI} / \mathrm{r}$ combination as compared to baseline (Figure $2 \mathrm{C}$ and Figure S4B). At the genus level, Butyricicoccus ( $\log _{2}$ fold change $-1.09, P=0.007)$, Lactobacillus ( $\log _{2}$ fold change $-5.57, \mathrm{P}=$ 0.007 ), Lachnospira ( $\log _{2}$ fold change $\left.-2.77, P=0.04\right)$, Dorea ( $\log _{2}$ fold change $-0.83, P=0.016$ ), and Prevotella ( $\log _{2}$ fold change $-2.00, P=0.007$ ) exhibited a significant curtailment in abundance after the PI-based treatment ( $2 \mathrm{NRTI}+\mathrm{PI} / \mathrm{r}$ ) (Figure 2D and Figure S4B). In addition, when we compared the fold changes (after therapy as compared to before therapy) of different bacterial taxa between the two treatment regimens (NNRTI v/s PI), the mean difference led to the outcome of 

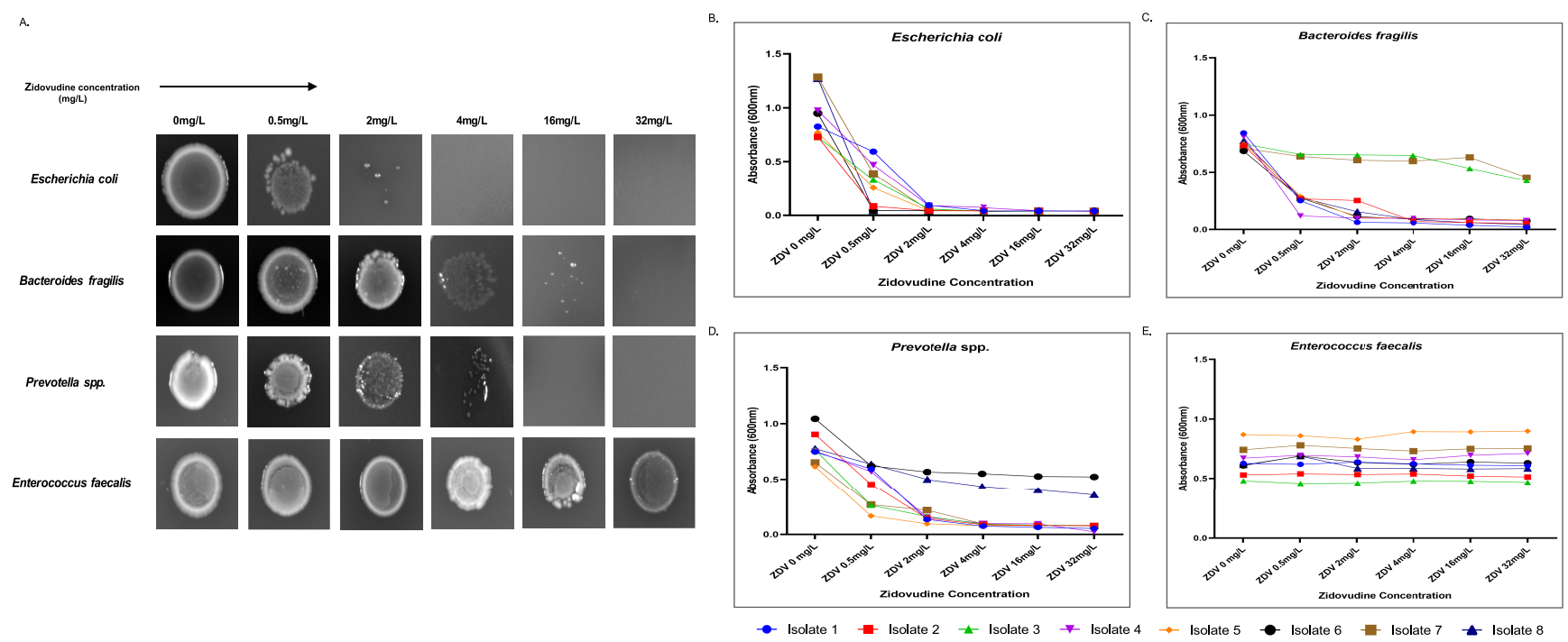

Figure 3. Microbial susceptibility assay against zidovudine (ZDV). (A) Agar dilution assay: brucella agar plate images of E. coli, Bacteroides fragilis, Prevotella spp., and Enterococcus faecalis with an increasing amount of ZDV at $0.5,2,4,16$, and $32 \mathrm{mg} / \mathrm{L}$. (B-E) Broth dilution assay: growth curves of E. coli, Bacteroides fragilis, Prevotella spp., and Enterococcus faecalis against ZDV at $0.5,2,4,16$, and $32 \mathrm{mg} / \mathrm{L}$. Growth was measured by monitoring the absorbance at $600 \mathrm{~nm}$. Data represent the average of three independent determinations.

three gut microbes, namely, Lactobacillus $(P=0.014)$, Dorea $(P$ $=0.007)$, and Butyricimonas $(P=0.03)$, which is represented in Figure S5 in the Supporting Information.

Zidovudine shows Antibacterial Activity against Bacteroides and Prevotella. To investigate the microbiota changes under various ART regimens, we experimented the in vitro effect of 15 antiretroviral drugs individually against the predominant gut microbes, namely, Escherichia coli, Enterococcus faecalis, Bacteroides fragilis, and Prevotella spp. Antimicrobial susceptibility was tested via agar dilution assay and broth dilution assay. Among the different NRTIs tested, zidovudine (ZDV) showed antibacterial activity within a defined range of concentrations. E. coli was previously shown to be inhibited by $\mathrm{ZDV}$ and hence was used as a positive control. ${ }^{29} \mathrm{ZDV}$ had an MIC of $0.5-1 \mathrm{mg} / \mathrm{L}$ against $E$. coli, $4-8 \mathrm{mg} / \mathrm{L}$ against Bacteroides fragilis, and $4 \mathrm{mg} / \mathrm{L}$ against Prevotella spp. (Figure 3A). A marked decrease in inoculum size was observed for the susceptible microbes under specific drug concentrations, thereby corroborating with their respective MIC values (Figure $3 \mathrm{~A}$ ). The broth dilution assay showed better sensitivity, leading to similar MIC values: $0.5 \mathrm{mg} / \mathrm{L}$ for $E$. coli, $2-4 \mathrm{mg} / \mathrm{L}$ for Prevotella spp., and $0.5-2 \mathrm{mg} / \mathrm{L}$ for $B$. fragilis (Figure $3 \mathrm{~B}-\mathrm{D}$ ). E. faecalis however did not show any growth inhibition under the effect of ZDV, which is also evident by the stability of its growth curve $(600 \mathrm{~nm})$ under different ZDV concentrations (Figure 3E). Approximately $100 \%$ of E. coli isolates, $70 \%$ of Prevotella spp. isolates, and $75 \%$ of Bacteroides spp. isolates were susceptible to ZDV. At the highest concentrations of ZDV, isolates of Prevotella spp. and Bacteroides spp. showed prominent growth inhibition. Table 1 presents the effectivity of zidovudine as an antimicrobial agent against the isolates of the abovementioned microbes.

Abacavir (ABC), Emtricitabine (FTC), Lamivudine (3TC), Tenofovir (TFV), Tenofovir disoproxil fumarate (TFD), and Tenofovir Alafenamide (TAF) did not show any sign of growth inhibition against tested isolates (Table 2). However, Stavudine (d4T) showed antimicrobial activity against one isolate of Bacteroides spp. and two isolates of Prevotella spp. with a high $\mathrm{MIC}$ of $128 \mathrm{mg} / \mathrm{L}$. To exclude the temperature effect (at $42{ }^{\circ} \mathrm{C}$,
Table 1. Antimicrobial Activity of Zidovudine and Efavirenz Tested against 35 Isolates of E. coli, 20 Isolates of E. faecalis, 22 Isolates of Bacteroides spp., and 10 Isolates of Prevotella spp. by Broth Dilution Method

\begin{tabular}{lccc}
\multicolumn{4}{c}{ antibacterial effect of Zidovudine } \\
\hline \multicolumn{1}{c}{ strains } & ${ }^{a} \mathrm{MIC}_{50}(\mathrm{mg} / \mathrm{L})$ & ${ }^{b} \mathrm{MIC}_{90}(\mathrm{mg} / \mathrm{L})$ & susceptibility (\%) \\
E. coli & 0.5 & 1 & 100 \\
Bacteroides spp. & 4 & 8 & 75 \\
Prevotella spp. & 4 & 8 & 70 \\
E. faecalis & $>128$ & $>128$ \\
\multicolumn{4}{c}{ antibacterial effect of Efavirenz } \\
\hline E. coli & $>256$ & $>256$ & 0 \\
Bacteroides spp. & 16 & 128 & 70 \\
Prevotella spp. & 16 & 32 & 100 \\
E. faecalis & 16 & 32 & 100
\end{tabular}

${ }^{a} \mathrm{MIC}_{50}$ and ${ }^{b} \mathrm{MIC}_{90}$ are defined as the minimal concentration of drug capable of inhibiting $50 \%$ and $90 \%$ of the isolates tested, respectively.

which is used for preparation of agar plates) on the drug activity, we performed the drug sensitivity assay, which did not affect the antiviral activity of the drugs (Figure S4).

Efavirenz Inhibits Growth of Enterococcus, Bacteroides, and Prevotella. Additionally, we investigated the effect of NNRTIs, i.e., Nevirapine (NVP), Rilpivirine (RPV), and Efavirenz (EFV) on bacterial growth. EFV showed a consistent MIC of $32 \mathrm{mg} / \mathrm{L}$ against E. faecalis, $32 \mathrm{mg} / \mathrm{L}$ for Prevotella spp., and $32-128 \mathrm{mg} / \mathrm{L}$ against Bacteroides spp. (Figure 4A). No growth susceptibility was observed in $E$. coli against any concentration of EFV (Figure 4A,B). The effect of EFV resulted in a reduced growth phase, thereby producing progressively smaller inoculum density (Figure 4A) and validating their respective MIC values. The MIC values from the broth dilution assay were observed to be 16, 32, and $32 \mathrm{mg} / \mathrm{L}$ for Prevotella spp., Bacteroides spp., and E. faecalis, respectively (Figure 3CE). The highest concentration of EFV interestingly resulted in $100 \%$ growth inhibition of Prevotella spp. isolates, $100 \%$ of Enterococcus faecalis isolates, and $75 \%$ of Bacteroides spp. isolates (Figure 4C-E). However, NVP and RPV did not show any 
Table 2. Minimum Inhibitory Concentrations ${ }^{a}$ (MIC) of 15 Antiretorovirals and 9 Combinatorial Treatment against E. coli, Bacteroides spp., Prevotella spp., and E. faecalis

\begin{tabular}{|c|c|c|c|c|c|}
\hline \multirow[t]{2}{*}{ compound } & \multirow[t]{2}{*}{ abbreviation } & \multicolumn{4}{|c|}{$\mathrm{MIC}(\mathrm{mg} / \mathrm{L})$} \\
\hline & & E. coli & Bacteroides spp. & Prevotella spp. & E. faecalis \\
\hline \multicolumn{6}{|c|}{ non-nucleoside reverse transcriptase inhibitors } \\
\hline Efavirenz & EFV & $>256$ & $32-128$ & 32 & $16-32$ \\
\hline Nevirapine & NVP & $>256$ & $>256$ & $>256$ & $>256$ \\
\hline Rilpivirine & RPV & $>128$ & $>128$ & $>128$ & $>128$ \\
\hline \multicolumn{6}{|c|}{ nucleoside reverse-transcriptase inhibitors } \\
\hline Abacavir & $\mathrm{ABC}$ & $>256$ & $>256$ & $>256$ & $>256$ \\
\hline Emtricitabine & FTC & $>128$ & $>128$ & $>128$ & $>128$ \\
\hline Lamivudine & $3 \mathrm{TC}$ & $>128$ & $>128$ & $>128$ & $>128$ \\
\hline Stavudine & $\mathrm{d} 4 \mathrm{~T}$ & $>256$ & $>256$ & $>256$ & $>256$ \\
\hline Zidovudine & ZDV & $0.5-1$ & $4-8$ & 4 & $>128$ \\
\hline Tenofovir & TFV & $>256$ & $>256$ & $>256$ & $>256$ \\
\hline Tenofovir disoproxil fumarate & TDF & $>128$ & $>128$ & $>128$ & $>128$ \\
\hline Tenofovir Alafenamide & TAF & $>128$ & $>128$ & $>128$ & $>128$ \\
\hline \multicolumn{6}{|c|}{ protease inhibitors } \\
\hline Atazanavir & ATV & $>256$ & $>256$ & $>256$ & $>256$ \\
\hline Darunavir & DRV & $>512$ & $>512$ & $>512$ & $>512$ \\
\hline Lopinavir & LPV & $>128$ & $>128$ & $>128$ & $>128$ \\
\hline Ritonavir & RTV & $>128$ & $>128$ & $>128$ & $>128$ \\
\hline \multicolumn{6}{|c|}{ combinations } \\
\hline $\mathrm{EFV} / \mathrm{FTC} / \mathrm{TDF}$ & & $>240: 100: 120$ & $30: 12: 15$ & $15: 6: 7.5$ & $30: 12: 15$ \\
\hline 3TC/ZDV/EFV & & $30: 25: 60$ & $15: 12: 30$ & $15: 12: 30$ & $30: 25: 60$ \\
\hline $\mathrm{EFV} / \mathrm{ABC} / 3 \mathrm{TC}$ & & $>240: 240: 120$ & $30: 30: 15$ & $30: 30: 15$ & $30: 30: 15$ \\
\hline TDF/FTC/RPV & & $>120: 100: 80$ & $>120: 100: 80$ & $>120: 100: 80$ & $>120: 100: 80$ \\
\hline TDF/FTC/NVP & & $>120: 100: 160$ & $>120: 100: 160$ & $>120: 100: 160$ & $>120: 100: 160$ \\
\hline $3 \mathrm{TC} / \mathrm{ABC} / \mathrm{DRV}$ & & $>120: 240: 320$ & $>120: 240: 320$ & $>120: 240: 320$ & $>120: 240: 320$ \\
\hline $3 \mathrm{TC} / \mathrm{ABC} / \mathrm{LPV}$ & & $>120: 240: 120$ & $>120: 240: 120$ & $>120: 240: 120$ & $>120: 240: 120$ \\
\hline TDF/FTC/LPV & & $>120: 100: 120$ & $>120: 100: 120$ & $>120: 100: 120$ & $>120: 100: 120$ \\
\hline TDF/FTC/DRV & & $>120: 100: 320$ & $>120: 100: 320$ & $>120: 100: 320$ & $>120: 100: 320$ \\
\hline
\end{tabular}
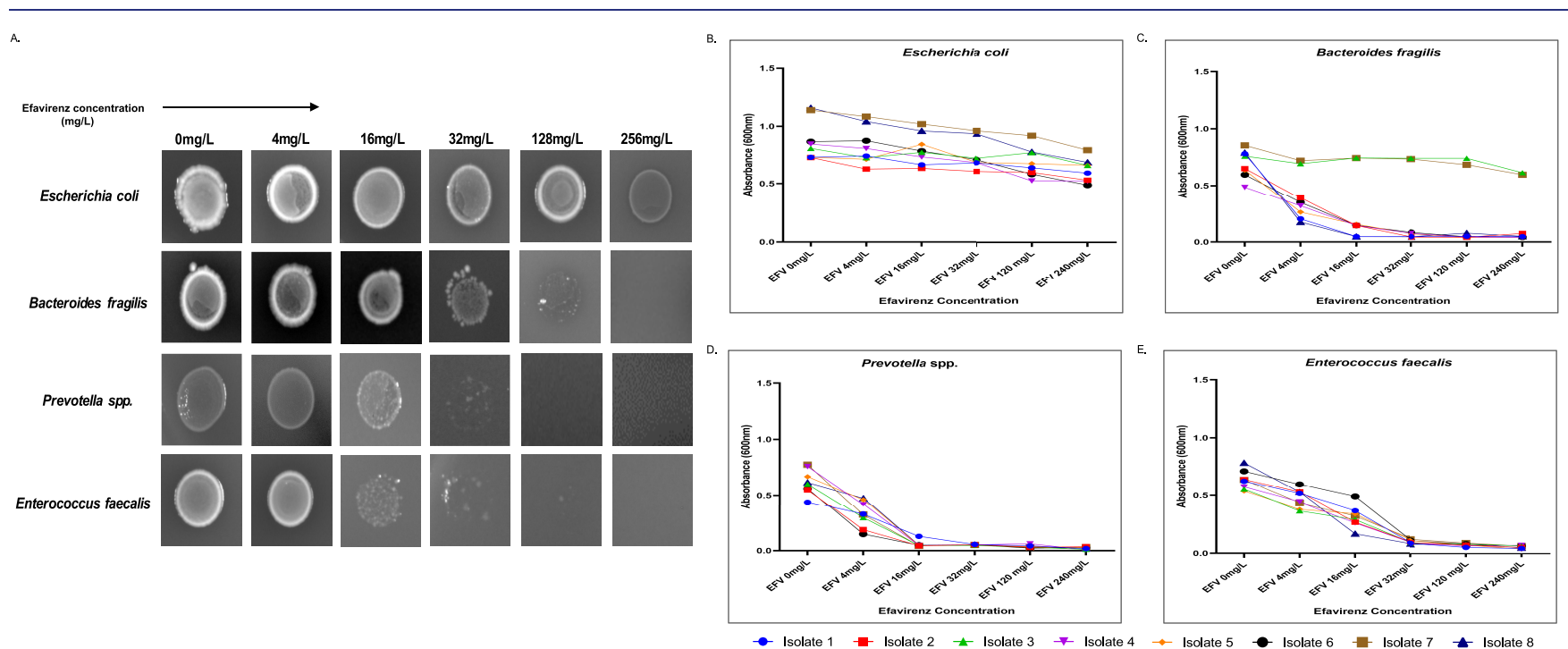

Figure 4. Microbial susceptibility assay against efavirenz (EFV). (A) Agar dilution assay: brucella agar plate images of E. coli, Bacteroides fragilis, Prevotella spp., and Enterococcus faecalis with an increasing amount of EFV at 4, 16, 32, 128, and $256 \mathrm{mg} / \mathrm{L}$. (B-E) Broth dilution assay: growth curves of E. coli, Bacteroides fragilis, Prevotella spp., and Enterococcus faecalis against EFV at 4, 16, 32, 128, and $256 \mathrm{mg} / \mathrm{L}$. Growth was measured by monitoring the $\mathrm{OD}$ at $600 \mathrm{~nm}$. Data represent the average of three independent determinations.

antibacterial activity under these similar testing conditions. The $\mathrm{MIC}_{50}$ and $\mathrm{MIC}_{90}$ of Efavirenz against the tested microbes depicted that EFV had a pronounced effect on the growth inhibition of E. faecalis and Prevotella spp. as compared to that of Bacteroides spp. (Table 1).

Absence of Microbial Susceptibility against Protease

Inhibitors. Different concentrations of Atazanavir (ATV), 
Darunavir (DRV), Lopinavir (LPV), and Ritonavir (RTV) were tested against the four gut microbes (E. coli, E. faecalis, Bacteroides fragilis, and Prevotella spp.). No signs of microbial growth inhibitory effect were observed under the action of the above-mentioned protease inhibitors, even at the highest concentrations (Table 2).

Effect of Combination ART on Gut Microbes. Among the nine tested drug combinations (Table 2), the combinations containing EFV (with FTC and TDF or 3TC and ABC) showed antibacterial effects against E. faecalis, Bacteroides, and Prevotella spp. and resistance against $E$. coli. In contrast, the combinatorial mix of EFV with 3TC and ZDV exerted growth inhibitory effects on all the four gut microbes tested, E. coli, E. faecalis, Bacteroides, and Prevotella spp. (Table 2). The MIC values were not indicative of any synergistic or antagonistic effect of the combined drugs.

\section{DISCUSSION}

In the present study, we report the in vitro antibacterial activity of antiretrovirals against Gram-positive and Gram-negative bacteria. Our results clearly show that zidovudine and efavirenz can affect the gut microbiota through their antimicrobial activity. In light of this, first, we proved that zidovudine had broad antibacterial activity against isolates of Escherichia coli, Bacteroides, and Prevotella species. Second, our data demonstrated that EFV inhibits growth of three bacterial taxa, namely, Enterococcus faecalis, Prevotella spp., and Bacteroides spp. Moreover, the microbiome analysis indicates that there is a specific microbial signature associated with NNRTI versus PI treatment.

Studies conducted during the early times of the HIV epidemic reported zidovudine to have a bactericidal effect against $E$. coli and some other members of Enterobacteriaceae family like $S$. typhimurium and Shigella flexneri. ${ }^{29}$ Specifically, ZDV affects bacterial growth through DNA chain termination and requires phosphorylation by cellular kinases present in bacteria for activation. Previous studies, in fact, have shown that the absence of or mutations in the thymidine kinase (phosphokinase) can overcome ZDV effect and might also cause ZDV resistance. Thus, in a study of ZDV treated HIV-1-infected individuals, 6 out 11 fecal samples harbored ZDV-resistant E. coli. ${ }^{30}$ Here, we have not only confirmed that ZDV inhibits E. coli (MIC of $0.5-1$ $\mathrm{mg} / \mathrm{L}$ ) but also reported the novel findings of the antibacterial effect of ZDV on Bacteroides spp. and Prevotella spp. with a low MIC of $4-8 \mathrm{mg} / \mathrm{L}$. These in vitro findings presume an in vivo effect of ZDV on the bacterial content of the gut lumen, knowing that the predicted gut concentration of drugs is much higher. ZDV is one of the oldest antiretrovirals but it is still a part of cART (combination antiretroviral therapy) especially in lowincome countries. Moreover, in high-income countries like Sweden, ZDV is used during delivery and the first weeks of postpartum.

The major microbial populations inhabiting the large intestine are Bifiobacterium, Bacteroides, Lactobacillus, Clostridium, Fusobacterium, and Enterobacteria. We can speculate that the use of the ZDV can have profound consequences on gut microbiome, affecting both Enterobacteriaceae and Bacteroides. For example, Escherichia coli belonging to Enterobacteriaceae is the first microbe to colonize infants and is abundantly present in the human gut ${ }^{31}$ (constituting about $0.1 \%$ ), existing in a symbiotic relationship to benefit the humans in metabolism and vitamin $\mathrm{K}$ production, providing additional nutrients and enabling improved acquisition and preventing the colonization by pathogens as well providing resistance against them. ${ }^{32}$ Changes in E. coli composition is associated with human health status since it is basically linked to the different strains of E. coli (commensal or pathogenic) harboring the gut and their pathogenic potential. ${ }^{33,34}$ E. coli is associated with differences in gut diets and stress-related gene expression, which are basically linked to obesity and diabetes. ${ }^{35}$

Additionally, EFV also showed broad antibacterial properties against both Gram-positive (Enterococcus faecalis) and Gramnegative (Prevotella spp. and Bacteroides spp.) isolates. The reported MIC values of EFV were 16, 32, and $32 \mathrm{mg} / \mathrm{L}$ for Prevotella spp., Bacteroides spp., and E. faecalis, respectively. Although, the EFV MIC values were higher than the ZDV ones, still both the estimated and reported gut concentrations of EFV were much higher than the MIC values reported in our study. ${ }^{36-38}$ Adding to our findings, a recent study by Shilaih et al. ${ }^{37}$ reported the antibacterial effect of EFV on Bacillus subtilis with an MIC of $16 \mathrm{mg} / \mathrm{L}$. EFV is yet one of most prescribed antivirals worldwide, and it is estimated that several millions individuals are prescribed this drug on a daily basis. ${ }^{39-42}$ Interestingly, EFV has been known to have additional effects, aside from its specified antiviral efficacy. EFV, in fact, has been reported to have a cytotoxic effect on tumor cells through the induction of oxidative stress. ${ }^{43}$ Moreover, it has also been shown to exert a strong anti-plasmodial activity in vitro ${ }^{44}$ as well as a similar effect on Leishmania spp. ${ }^{45}$ On the contrary, to our knowledge, the bactericidal effect of EFV reported in this study has never been previously observed. This is the first study to demonstrate that efavirenz has antibacterial properties against three bacterial species, namely, Enterococcus faecalis, Prevotella spp., and Bacteroides spp. These members of the gut microbiota might be responsible for health benefits or disorders, depending on species alteration after a dysbiotic event. E. faecalis is an early gut colonizer contributing to gut homeostasis, early intestinal immune development, and regulation of inflammatory responses via PPAR $\gamma 1$-induced IL-10 and TGF- $\beta$ expression in vitro and is capable of reducing the severity of diarrhea in the younger population. Additionally, this bacterium also attenuates the expression of proinflammatory cytokines like IL- 8 and has hence been known to have potential probiotic activity. Notably, enterococcal strains, which comprise $1 \%$ of intestinal microbiota, with E. faecalis and E. faecium being the more represented members, are commensal bacteria capable of eliminating the exogenous and pathogenic enterococcal competitors from the gut thanks to their sophisticated defense systems. The changes in E. faecalis populations caused by efavirenz treatment have the potential to modify the genes related to its virulence, biofilm production, and antibiotic resistance. Similarly, the Bacteroidetes phylum comprises Bacteroides and Prevotella as the predominant obligate anaerobes, with Bacteroides establishing balanced, long-term association with their hosts for many health benefits. ${ }^{46,47}$ As commensals and mutualists, Bacteroidetes members are prominent polysaccharide degraders and benefit individuals with dysbiotic gut bacteria. For example, Bacteroides fragilis produces a polysaccharide that enables the immune system development in mice with Th1/Th2 balances in lymphoid tissues as well as contributing to efficient systemic T-cell functioning. Moreover, in the last decades, Bacteroides spp. have been inversely correlated with obesity, highlighting the importance of the carbohydrate metabolism for this disease. Conversely, Prevotella members are crucial for mucin degradation, which could cause increased epithelial permeability. Notably, however, higher numbers of Prevotella copri are 
reported to be drivers of inflammation in HIV-infected individuals and in rheumatoid arthritis patients. On the contrary, Prevotella histicola is known to induce anti-inflammatory signals in a murine arthritis model, suggesting that different members of this genus might have different immunomodulating capabilities.

In summary, we showed that ART containing ZDV or EFV exerted antibacterial effect, although any synergistic impact was not evident. Conversely, several other antiretrovirals showed no effect on the bacterial growth in our model. However, it is possible that the lack of antibacterial effect is due to low dosage as the highest concentrations tested have been guided by the previous pharmacokinetic studies. ${ }^{48}$ Alternatively, the antiretrovirals might have undergone active metabolism by some specific components of microbiome. This, for example, has been shown for TDF, which is metabolized by Gardnerella vaginalis but not by Lactobacillus spp. (both present in the vaginal microbiome) and hence could explain the low efficacy of topical TDF as pre-exposure prophylaxis, as seen in the CAPRISA trial. $^{49}$ Significant perturbations in the complex and fragile balance of the intestinal microbiota could lead to disruption of the symbiotic relationships with our commensals, causing health complications. In light of this, our results prompt the question of how these microbes are affected by the two antivirals described in this study (ZDV and EFV), with which molecular mechanisms, and whether these antiretrovirals can be used as potential antibacterials against them. Future analyses on this topic might hopefully offer an alternative to the widely used antibiotic-centered treatments for diseases and disorders whose etiopathogenesis involves these bacterial species, as antibiotic resistance in bacteria grows exponentially each year and it is establishing itself as a global threat to human health.

The microbiome analysis of our longitudinal cohort also showed that bacterial communities after the antiretroviral exposure were less diverse and distinct as compared to those before ART. Differential changes in the gut microbiome $\alpha$ diversity were observed between various ART treatments, where NNRTI-based therapy (2 NRTI+NNRTI) showed distinct reduction in bacterial diversity and richness significantly, whereas PI-based treatment $(2 \mathrm{NRTI}+\mathrm{PI} / \mathrm{r})$ did not show a similar trend. Moreover, we report specific bacterial signatures associated with treatment where several taxa were affected by ART. Among the genera affected, Dorea, a major constituent of healthy gut flora, showed reduced abundance in patients treated with PI-based therapy. This bacterium is also linked to proinflammation in IBD patients. Additionally, Lachnospira and Oscillospira, the short chain fatty-acid producers showed reduced abundance in patients after ART (Figure S2C), and this might possibly affect the decrease in HIV-induced inflammation in the same individuals. ${ }^{50,51}$ By stratifying the cohort into the usage of NNRTI ( $80 \% \mathrm{EFV})$, we could reveal that the effect on Bacteroidetes (family Prevotellaceae) was more evident in this group as substantiated by the in vitro data. However, even patients treated with PI showed a decrease in Prevotellaceae, and hence, the different microbiome patterns in both treatment groups suggest a more complex mechanism of ART interaction with bacteria.

The bacterial communities colonizing various regions of the human gut play specific roles in host metabolism, immunomodulation, and different health aspects. A standard healthy optimal gut microbiome profile is far from existing in this research world since it is different for every individual; however, a healthy balance between the host and microbial community can be maintained for effective metabolic and immune functioning to prevent infections. Hence, our study aimed to reflect the changes in the microbiota composition due to antiretrovirals, which can be studied in subsequent years to modulate the treatment strategies for HIV infection. Studies on the short- and long-term impact of ART on the gut microbiome have repeatedly shown that ART alone is unable to restore gut dysbiosis and inflammation. ${ }^{22}$ Thus, the gut microbiome in ART treated patients displays different community structures as opposed to those of untreated HIV patients and healthy individuals. Findings of decreased $\alpha$-diversity in the microbiome community is present in nearly all studies; albeit the compositional taxa differences are in general study bound. ${ }^{12,52,53}$ Like our study, Sortino et al. also found a lower proportion of Bacteroidetes and a higher Proteobacteria post-ART as compared to pre-ART. ${ }^{54}$ Also, a study from a Nigerian cohort reported decreased abundance of microbes belonging to phylum Bacteroidetes in ART treated patients. ${ }^{20}$ Moreover, studies from Spain $^{55}$ and Mexico ${ }^{22}$ have found increased Proteobacteria and differences in Ruminococcaceae family, respectively, between ART (PI and NNRTI) and controls. However, the interpretation of the findings from different studies is challenging as most of them are cross-sectional and the results are conflicting in the sampling/methodology used. A comparative study of PI, NNRTI, and integrase inhibitor-based ART showed integrase inhibitors to be most effective against host inflammation while displaying minor modulation of gut composition. ${ }^{55}$ Although this study was cross-sectional, several other reports have shown that different ARTs show differential effect on gut inflammation. ${ }^{23,52,56}$ Also, considering results from our study, it is sensible to speculate that ART alone might not be capable of restoring the damaged gut-blood barrier in chronic HIV, and therefore, the antiretroviral interaction with the microbiome should be incorporated in treatment strategies. Such strategies should include microbiome modulation by pro- and prebiotics supplementation or fecal microbiota transplantation (FMT). Until now, in fact, several probiotics trials have been conducted showing minor/moderate effect on immune reconstitution and systemic inflammation in HIV infection. ${ }^{57-60}$ The preclinical evaluation for FMT efficiency in ART treated SIV-infected rhesus macaques, for example, showed increased peripheral Th17 and Th22 cell frequencies and reduced activation of gut CD4+ T-cells post-FMT. ${ }^{61}$ Additionally, in a small trial of HIVinfected patients, partial engraftment of the microbiome was achieved 8 weeks after FMT with a limited effect on the inflammatory markers. ${ }^{62}$

The biggest strength of this study is the novelty of the approach and the longitudinal design of the cohort. Our investigation, in fact, tackles the newborn field of the antimicrobial effects of antiviral drugs, which is garnering an increasing amount of attention in recent years. Though our clinical cohort had a limitation of low sample size under different treatments, we however dissect the antibacterial properties of several ARVs in this study, primarily focusing on the most commonly prescribed drugs in low-income countries (ZDV, $\mathrm{EFV}$ ). In summary, our results shed light on the alteration of the microbiome in HIV-infected individuals and show that one year of successful ART therapy does not revert the microbiota to the state prior to the infection. Moreover, antiretroviral drugs can induce alterations in the microbiome, both in its diversity and richness, due to their antibacterial properties, as demonstrated in-depth for ZDV and EFV. The knowledge that, worldwide, 30 million individuals with HIV are on ART daily, ${ }^{63,64}$ highlights the need to investigate all the potentially detrimental effects that 
antiretroviral drugs might have on patients under ART regimens, such as the induction of gut dysbiosis. Our analyses pave the way to additional studies investigating antiretroviral drugs and their bactericidal effects, potentially on an even broader scale, to determine the supplementary therapy needed to prevent or limit ART-induced microbiome alterations in HIV-infected patients. Moreover, considering the ever-growing health concern caused by antibiotic resistant bacteria, it is tempting to look at ARTs as a potential alternative to antibiotics, prompting more studies to investigate the feasibility of such a therapy.

\section{CONCLUSION}

Gut microbiota dysbiosis has been associated with HIV pathogenesis and these multifactorial gut alterations are coupled with key markers of host inflammation and immune activation. However, it is still unclear how these intestinal microbial alterations are affected by ART drugs administration. While some studies state the non-restoration of gut microflora to normal conditions under ART, others report dramatic outcomes for the further deterioration of the gut microbiome's equilibrium after ART, due to drug metabolization by bacteria or to the antimicrobial properties of antiretrovirals. Though specific, in fact, antiretrovirals have still been shown to possess antibacterial, antifungal, and anticancer activities. Therefore, in summary, the effect of antiretrovirals on microbial communities leading to gut modification during HIV treatment has been the major question, which we have tried to address in our present study. To do this, we (1) investigated the $16 \mathrm{~S}$ rRNA profiles from the fecal samples of untreated HIV-infected subjects and ART treated individuals under two different regimens (2 NRTI+NNRTI and 2 NRTI $+\mathrm{PI} / \mathrm{r}$ ) and (2) further evaluated the in vitro antibacterial effect of antiretrovirals to explore the differential microbial landscape under different ARTs. Notably, this has led us to discover the antibacterial activity of efavirenz (EFV), here reported for the first time. This finding further frames our results in a larger perspective, since EFV is a key antiretroviral used extensively in low-income countries. Additionally, our results depict the antimicrobial activity of zidovudine, which could potentially lead to further gut modification. In summary, we observed that ART does not restore the microbiome changes in HIV patients even after one year of effective treatment. Hence, these findings highlight the necessity to further investigate the gut microbiota in HIV patients under ART for better therapeutic interventions and gut health.

\section{METHODS}

Study Cohort. Sixteen subjects were included from a study on the gut microbiota and immune status in treatment-naive chronic HIV-1 patients followed for a median of 10 months (IQR 4-15) after ART initiation. ${ }^{8}$ The patients were chosen due to the availability of $16 \mathrm{~S}$ rRNA sequences for further analyses at baseline (before ART) and at follow up. ART comprised two NRTIs (nucleoside reverse transcriptase inhibitor) in combination with a non-nucleoside reverse transcriptase inhibitor (NNRTI; $n=8$ ) or a ritonavir-boosted protease inhibitor $(\mathrm{PI} / \mathrm{r} ; n=8)$. The study participants were negative for antibiotics or probiotic consumption (two months prior sampling). The Regional Ethical Committee had approved the study (EPN Stockholm, Sweden, Dnr 2009-1485-31-3).

Sequence Analysis. This is a longitudinal study where the HIV-1 individuals were followed up for 10 months after ART administration and, hence, were categorized into two groups as before ART $(n=16)$ and after ART $(n=16)$. After ART introduction, the participants were further divided into subgroups based on the different ART regimens: 2 NRTI +NNRTI $(n=8)$ and 2 NRTI+PI/r $(n=8)$. 16S rRNA sequencing was performed on the Illumina MiSeq platform after DNA extraction from fecal samples as described earlier ${ }^{8}$ with modification in the sequence analysis pipeline. Afterward, reads were demultiplexed, preprocessed, and analyzed by QIIME. ${ }^{65} \alpha$ Diversity was determined by the diversity and richness indices such as observed: Chao1, ACE, Shannon, Simpson, and Fisher. Clustering of the samples was presented with a NMDS plot based on the Bray-Curtis distance, as compared to a principal coordinate analysis (PCoA) done previously. ${ }^{8}$ Both the $\alpha$ - and $\beta$-diversity were calculated with the phyloseq R package (version 1.26.1). The relative abundance of the samples was calculated using an in-house PERL script. Further, bar plots and box plots were constructed with the relative abundance values using the $R$ package ggplot2 (version 3.2.0 and $\mathrm{v}$ 3.3.2, respectively) to determine the differences in the microbial compositions between the two groups at the phylum, family, and genus levels. PERMANOVA is a non-parametric analysis used to test the differences in the microbial compositions between the two groups performed at the phylum level using the $\mathrm{R}$ package vegan (version R 2.5.5). The taxonomy tree of the microbial communities was visualized using GraPhlAn (Graphical Phylogenetic Analysis). ${ }^{66}$ The heatmap for determining the mean differences between treatments (NNRTIs v/s PIs) was constructed using the R package Complex Heatmap (2.2.0). For statistical analysis, Wilcoxon signed-rank test was performed.

Bacterial Isolates. Twenty isolates of Enterococcus faecalis, 35 isolates of Escherichia coli, 22 isolates of Bacteroides spp., and 10 isolates of Prevotella spp. were cultured from patient samples received at the Department of Clinical Microbiology, Karolinska University Hospital, Sweden. These microbes were isolated from deep wound secretions, intra-abdominal infections, or bloodstream infections with routine culture techniques and subsequent species identification with matrix-assisted laser desorption/ionization time-of-flight (MALDI-TOF) mass spectrometry (detection is based on the spectra of conserved ribosomal proteins), using a Microflex LT (Bruker Daltonics, Billerica, MA, USA). These identified clinical isolates were then tested for antimicrobial susceptibility in accordance to EUCAST guidelines. $^{67}$

Antiretroviral Agents. The antiretroviral drugs used for screening were zidovudine, emtricitabine, lamivudine, stavudine, tenofovir, tenofovir disoproxil fumarate, tenofovir alafenamide, efavirenz, rilpivirine, nevirapine, darunavir, atazanavir, lopinavir, and ritonavir. All these compounds were purchased from Selleckchem, Munich, Germany, except for lopinavir and nevirapine, which were procured from SigmaAldrich, St. Louis, MO, USA. These compounds were dissolved in their appropriate solutions (i.e., DMSO or water) before being used in the agar or broth dilution assays.

Gut Concentrations of Drugs. The maximum and minimum dosage for each drug were calculated on the estimated gut concentration and plasma concentration, respectively. The gut concentrations were assessed on the basis of the formulations published by the European Medicines Agency. ${ }^{48}$ The minimum concentration, $C_{\max }$, or maximum plasma concentration, were derived from fact sheets of HIV drugs. ${ }^{48}$ The different drug concentrations are outlined in Table 3. 
Table 3. Concentration of Antiretrovirals Used for Microbial Susceptibility Assay ${ }^{a}$

\begin{tabular}{|c|c|c|c|c|}
\hline drug name & $\begin{array}{l}\text { max drug } \\
\text { dose in one } \\
\text { occasion } \\
(\mathrm{mg})\end{array}$ & $\begin{array}{c}\text { concentration } \\
\text { in gut }{ }^{b} \\
(\mu \mathrm{g} / \mathrm{mL})\end{array}$ & $C_{\max }{ }^{c}(\mu \mathrm{g} / \mathrm{mL})$ & $50 \times C_{\max }{ }^{d}$ \\
\hline Efavirenz & 600 & 240 & 4.07 & 203.5 \\
\hline Nevirapine & 200 & 80 & 5.74 & 287 \\
\hline Rilpivirine & 150 & 60 & 0.204 & 10.2 \\
\hline Abacavir & 600 & 240 & 4.26 & 213 \\
\hline Emtricitabine & 200 & 80 & 1.8 & 90 \\
\hline Lamivudine & 300 & 120 & 2 & 100 \\
\hline Stavudine & 40 & 16 & 0.536 & 26.8 \\
\hline Zidovudine & 250 & 100 & 2.29 & 114.5 \\
\hline $\begin{array}{l}\text { Tenofovir } \\
\text { disoproxil } \\
\text { fumarate }\end{array}$ & 300 & 120 & 0.326 & 16.3 \\
\hline Tenofovir & 245 & 98 & 0.05 & 2.5 \\
\hline $\begin{array}{l}\text { Tenofovir } \\
\text { Alafenamide }\end{array}$ & 25 & 10 & 0.154 & 7.7 \\
\hline Atazanavir & 400 & 160 & 3.152 & 157.6 \\
\hline Darunavir & 800 & 320 & 6.5 & 325 \\
\hline Lopinavir & 800 & 320 & 9.6 & 480 \\
\hline Ritonavir & 100 & 40 & 0.85 & 42.5 \\
\hline \multicolumn{5}{|c|}{$\begin{array}{l}{ }^{a} \text { www.hivdrugnteractions.org. }{ }^{b} \text { Gut concentration }(\mathrm{mg} / \mathrm{L}) \text { was } \\
\text { assessed on the basis of the formulations published by the European } \\
\text { Medicines Agency. }{ }^{54}{ }^{c} C_{\max }: \text { maximum drug concentration in plasma. } \\
{ }^{d} 50 \times C_{\max } \text { : estimation of concentration for drugs. }\end{array}$} \\
\hline
\end{tabular}

Broth Microdilution Method. Broth dilution assay is required for the determination of the minimum inhibitory concentration (MIC) of microbes against antibiotics, also known as the lowest concentration of antibiotics that inhibits visible bacterial growth. In this experiment, we intended to investigate the susceptibility of microbes against antiretroviral agents. The samples were assayed in a 96-well plate with 2 -fold serial dilutions of the testing agent. Our compounds were tested at different concentrations depending on their respective gut concentrations; for instance, zidovudine was serially diluted 2fold, from 100 to $0.5 \mathrm{mg} / \mathrm{L}$ and efavirenz from 240 to $4 \mathrm{mg} / \mathrm{L}$. Stock solutions of all the compounds were prepared in appropriate solvents. The serial dilutions were carried out in small volumes of media in round-bottom 96-well plates. Specifically, Mueller Hinton Broth and Brucella broth ${ }^{68}$ (supplemented with $5 \%$ lysed horse blood, $5 \mu \mathrm{g} / \mathrm{mL}$ hemin, and $1 \mu \mathrm{g} / \mathrm{mL}$ Vitamin $\mathrm{K} 1$ (with respect to CLSI guidelines) ${ }^{67}$ were used for the assay of aerobic (E. coli and E. faecalis) and anaerobic bacteria (Bacteroides spp. and Prevotella spp.), respectively (Substrate department, Karolinska University Hospital, Solna). Overnight cultures of bacteria were diluted in a 1:100 ratio to have an initial inoculum of around $5 \times$ $10^{5} \sim 10^{6} \mathrm{CFU} / \mathrm{mL}$. The positive and negative controls were included, containing no drugs and no bacteria, respectively. Since the solvent concentration during the assay was around $2.5 \% \mathrm{v} / \mathrm{v}$, the solvents were also tested for their antibacterial activity at that particular concentration. Ampicillin, kanamycin, or chloramphenicol were used as the positive controls during the assay. GasPak sachets (Thermo Fisher Scientific), Anoxomat systems, or anaerobic jars were used for culturing anaerobes. ${ }^{69-73}$ The plates were incubated at $35{ }^{\circ} \mathrm{C}$ for $16-18 \mathrm{~h}$ for aerobes and 24-48 h for anaerobes prior to optical density measurement at $600 \mathrm{~nm}$ with an Infinite M200 spectrophotometer. The MIC was then determined from the lowest concentration of drug, showing $>90 \%$ bacterial killing relative to the control. This assay was repeated thrice in biological triplicates.

Agar Dilution Method. Agar dilution technique is the gold standard for MIC determination of anaerobic bacteria according to Clinical and Laboratory Standards Institute (CLSI) methodology. ${ }^{74}$ In this procedure, Brucella agar plates with $5 \%$ laked sheep blood, $5 \mathrm{mg} / \mathrm{L}$ hemin, and $1 \mathrm{mg} / \mathrm{L}$ Vitamin $\mathrm{K} 1$ are supplemented with different concentrations of antivirals. An initial inoculum of $0.5 \pm 0.1 \mathrm{McF}$ arland standard from pure bacterial cultures resuspended in $0.2 \% \mathrm{NaCl}$ was then cultured on these plates with the help of a sterile replicator device. The cultures were then incubated at $35^{\circ} \mathrm{C}$ for $16-18 \mathrm{~h}$ for aerobes and 24-48 $\mathrm{h}$ for anaerobes, following which the plates are compared visually (positive for lawn colonies and negative for no growth or single colony). On the basis of this method, the lowest antiviral concentration inhibiting microbial growth was determined. Though being labor intensive, this method is the most recommended for anaerobic work. ${ }^{75,76}$ A brucella agar plate without the drugs but only the bacterium was used as a positive control, while another plate supplemented with $4 \%$ DMSO was used as a control for toxicity.

In Vitro Drug Sensitivity Assay. TZM-bl cells and the pNL43 plasmid were obtained from the NIH AIDS Reagent Program. 293T cells were purchased from ATCC (Manassas, VA, USA). The cells were maintained in Dulbecco's modified eagle's medium (DMEM) (Sigma), supplemented with $10 \%$ fetal bovine serum and $2 \mathrm{mM}$ L-glutamine. The drug sensitivity assay enabled the determination of the ART activity to inhibit replication of the reference virus, NL43, in TZMbl cells. The antivirals were preheated at $42{ }^{\circ} \mathrm{C}$ prior to the assay to ensure their stable activity during supplementation in Brucella agar plates. The compounds at room temperature were taken as a positive control. The antivirals were serially diluted from 100 $\mu \mathrm{M}$ to $1.28 \mathrm{nM}$ and added in triplicate in a 96-well plate seeded with TZM-bl cells, followed by infection with a NL43 strain at an MOI of $0.05 \mathrm{IU} /$ cells in the presence of diethylaminoethyl (10 $\mathrm{mg} / \mathrm{mL}$ ). Virus replication was quantified by the measurement of luciferase activity (relative light units) using Bright-Glo luciferase assay system (Promega, Madison, WI, USA). Drug concentrations required for inhibiting virus replication by $50 \%$ $\left(\mathrm{IC}_{50}\right)$ were calculated with a dose-response curve using nonlinear regression analysis.

\section{ASSOCIATED CONTENT}

\section{Supporting Information}

The Supporting Information is available free of charge at https://pubs.acs.org/doi/10.1021/acsinfecdis.0c00536.

Figures of $\alpha$-diversity indices of gut microbiome, NMDS analysis, bar plots, taxonomic distribution of the microbial communities in the fecal samples of patients, PERMANOVA analysis of gut microbiota, heatmap depicting the level of significance between the fold changes of different bacterial taxa/genus in each individual patient, and antiretroviral drug activity under high-temperature conditions (PDF)

\section{Accession Codes}

The metadata and raw 16S rRNA gene sequence data generated and analyzed during this study are deposited at the NCBI SRA database (accession number pending). 


\section{AUTHOR INFORMATION}

\section{Corresponding Author}

Shilpa Ray - Department of Laboratory Medicine, Division of Clinical Microbiology, ANA Futura, Karolinska Institutet, Stockholm 141 52, Sweden; The Laboratory for Molecular Infection Medicine Sweden MIMS, Umeå University, Umeå 901 87, Sweden; 다이.org/0000-0002-9940-7366; Email: shilpa.ray@ki.se

\section{Authors}

Aswathy Narayanan - Department of Laboratory Medicine, Division of Clinical Microbiology, ANA Futura, Karolinska Institutet, Stockholm 141 52, Sweden

Christian G. Giske - Department of Laboratory Medicine, Division of Clinical Microbiology, ANA Futura, Karolinska Institutet, Stockholm 141 52, Sweden; Department of Clinical Microbiology, Karolinska University Hospital, Solna, Stockholm 171 76, Sweden

Ujjwal Neogi - Department of Laboratory Medicine, Division of Clinical Microbiology, ANA Futura, Karolinska Institutet, Stockholm 141 52, Sweden; (1) orcid.org/0000-0002-08443338

Anders Sönnerborg - Department of Laboratory Medicine, Division of Clinical Microbiology, ANA Futura, Karolinska Institutet, Stockholm 141 52, Sweden; Department of Medicine Huddinge, Division of Infectious Diseases, Karolinska University Hospital, Huddinge, Stockholm 141 86, Sweden

Piotr Nowak - Department of Laboratory Medicine, Division of Clinical Microbiology, ANA Futura, Karolinska Institutet, Stockholm 141 52, Sweden; The Laboratory for Molecular Infection Medicine Sweden MIMS, Umeå University, Umeå 901 87, Sweden; Department of Medicine Huddinge, Division of Infectious Diseases, Karolinska University Hospital, Huddinge, Stockholm 141 86, Sweden

Complete contact information is available at: https://pubs.acs.org/10.1021/acsinfecdis.0c00536

\section{Author Contributions}

Conceived and designed the experiments: S.R., P.N., and U.N. Coordinated the sample collection: P.N., C.G.G., and A.S. Performed the experiments: S.R. Analyzed the data: S.R., A.N., P.N., and U.N. Wrote the paper: S.R. and P.N. Reviewed and/or edited the manuscript: all authors

\section{Notes}

All procedures in this study were approved by the Regional Ethical Committee (EPN Stockholm, Sweden, Dnr 2009-148531-3).

The authors declare no competing financial interest.

\section{ACKNOWLEDGMENTS}

The authors acknowledge Shambhu Prasad Ganeshappa Aralaguppe and Duncan Njenda for their contribution in drug sensitivity assay. The study was supported by grants from Molecular Infection Medicine Sweden (MIMS, Umeå), Swedish Physicians Against AIDS research fund, Stockholm County Council (SLL-KI for PN; ALF nr 20190451), Swedish Research Council (Dnr 2016-01675; AS), Piotr Nowak is a MIMS Clinical Research Fellow.

\section{ABBREVIATIONS}

ART, antiretroviral therapy; HIV, human immunodeficiency virus; MIC, minimum inhibitory concentration; EFV, Efavirenz;
ZDV, Zidovudine; NRTI, nucleoside reverse transcriptase inhibitor; NNRTI, non-nucleoside reverse transcriptase inhibitor; PI, protease inhibitor; cART, combination antiretroviral therapy

\section{REFERENCES}

(1) Nicholson, J. K., Holmes, E., Kinross, J., Burcelin, R., Gibson, G., Jia, W., and Pettersson (2012) Host-gut microbiota metabolic interactions. Science 336 (6086), 1262-1267.

(2) Routy, B., Le Chatelier, E., Derosa, L., Duong, C. P., Alou, M. T. Daillère, R., Fluckiger, A., Messaoudene, M., Rauber, C., Roberti, M. P. J. S., et al. (2018) Gut microbiome influences efficacy of PD-1-based immunotherapy against epithelial tumors. Science 359 (6371), 91-97.

(3) Hill-Burns, E. M., Debelius, J. W., Morton, J. T., Wissemann, W. T., Lewis, M. R., Wallen, Z. D., Peddada, S. D., Factor, S. A., Molho, E., Zabetian, C. P., Knight, R., and Payami, H. (2017) Parkinson's disease and Parkinson's disease medications have distinct signatures of the gut microbiome. Mov. Disord. 32 (5), 739-749.

(4) Seo, D.-o., Boros, B. D., and Holtzman, D. M. (2019) The microbiome: A target for Alzheimer disease? Cell Res. 29 (10), 779780.

(5) Burcelin, R., Serino, M., Chabo, C., Blasco-Baque, V., and Amar, J. (2011) Gut microbiota and diabetes: from pathogenesis to therapeutic perspective. Acta Diabetol. 48 (4), 257-273.

(6) Rajca, S., Grondin, V., Louis, E., Vernier-Massouille, G., Grimaud, J.-C., Bouhnik, Y., Laharie, D., Dupas, J.-L., Pillant, H., Picon, L., Veyrac, M., Flamant, M., Savoye, G., Jian, R., Devos, M., Paintaud, G., Piver, E., Allez, M., Mary, J. Y., Sokol, H., Colombel, J.-F., and Seksik, P. (2014) Alterations in the intestinal microbiome (dysbiosis) as a predictor of relapse after infliximab withdrawal in Crohn's disease. Inflammatory Bowel Dis. 20 (6), 978-986.

(7) Lozupone, C. A., Li, M., Campbell, T. B., Flores, S. C., Linderman, D., Gebert, M. J., Knight, R., Fontenot, A. P., and Palmer, B. E. (2013) Alterations in the gut microbiota associated with HIV-1 infection. Cell Host Microbe 14 (3), 329-339.

(8) Nowak, P., Troseid, M., Avershina, E., Barqasho, B., Neogi, U., Holm, K., Hov, J. R., Noyan, K., Vesterbacka, J., Svärd, J., Rudi, K., and Sönnerborg, A. (2015) Gut microbiota diversity predicts immune status in HIV-1 infection. AIDS 29 (18), 2409-2418.

(9) Vesterbacka, J., Rivera, J., Noyan, K., Parera, M., Neogi, U., Calle, M., Paredes, R., Sönnerborg, A., Noguera-Julian, M., and Nowak, P. (2017) Richer gut microbiota with distinct metabolic profile in HIV infected Elite Controllers. Sci. Rep. 7 (1), 1-13.

(10) Guadalupe, M., Reay, E., Sankaran, S., Prindiville, T., Flamm, J., McNeil, A., and Dandekar, S. (2003) Severe CD4+ T-cell depletion in gut lymphoid tissue during primary human immunodeficiency virus type 1 infection and substantial delay in restoration following highly active antiretroviral therapy. J. Virol. 77 (21), 11708-11717.

(11) Dillon, S. M., Frank, D. N., and Wilson, C. C. (2016) The gut microbiome and HIV-1 pathogenesis: a two way street. AIDS 30 (18), 2737.

(12) Liu, J., Williams, B., Frank, D., Dillon, S. M., Wilson, C. C., and Landay, A. L. (2017) Inside out: HIV, the gut microbiome, and the mucosal immune system. J. Immunol. 198 (2), 605-614.

(13) Serrano-Villar, S., Vázquez-Castellanos, J. F., Vallejo, A., Latorre, A., Sainz, T., Ferrando-Martínez, S., Rojo, D., Martínez-Botas, J., del Romero, J., Madrid, N., Leal, M., Mosele, J. I., Motilva, M. J., Barbas, C., Ferrer, M., Moya, A., Moreno, S., Gosalbes, M. J., and Estrada, V. (2017) The effects of prebiotics on microbial dysbiosis, butyrate production and immunity in HIV-infected subjects. Mucosal Immunol. $10(5), 1279$

(14) Pérez-Santiago, J., Gianella, S., Massanella, M., Spina, C. A., Karris, M. Y., Var, S. R., Patel, D., Jordan, P. S., Young, J. A., Little, S. J., Richman, D. D., and Smith, D. M. (2013) Gut Lactobacillales are associated with higher $\mathrm{CD} 4$ and less microbial translocation during HIV infection. AIDS 27 (12), 1921.

(15) Dinh, D. M., Volpe, G. E., Duffalo, C., Bhalchandra, S., Tai, A. K., Kane, A. V., Wanke, C. A., and Ward, H. D. (2015) Intestinal 
microbiota, microbial translocation, and systemic inflammation in chronic HIV infection. J. Infect. Dis. 211 (1), 19-27.

(16) Dillon, S., Lee, E., Kotter, C., Austin, G., Dong, Z., Hecht, D., Gianella, S., Siewe, B., Smith, D., Landay, A. L., Robertson, C. E., Frank, D. N., and Wilson, C. C. (2014) An altered intestinal mucosal microbiome in HIV-1 infection is associated with mucosal and systemic immune activation and endotoxemia. Mucosal Immunol. 7 (4), 983.

(17) De Filippo, C., Cavalieri, D., Di Paola, M., Ramazzotti, M., Poullet, J. B., Massart, S., Collini, S., Pieraccini, G., and Lionetti, P. (2010) Impact of diet in shaping gut microbiota revealed by a comparative study in children from Europe and rural Africa. Proc. Natl. Acad. Sci. U. S. A. 107 (33), 14691-14696.

(18) Dillon, S., Lee, E., Kotter, C., Austin, G., Gianella, S., Siewe, B., Smith, D., Landay, A., McManus, M., Robertson, C. E., Frank, D. N., McCarter, M. D., and Wilson, C. C. (2016) Gut dendritic cell activation links an altered colonic microbiome to mucosal and systemic T-cell activation in untreated HIV-1 infection. Mucosal Immunol. 9 (1), 2437.

(19) Troy, E. B., and Kasper, D. L. (2010) Beneficial effects of Bacteroides fragilis polysaccharides on the immune system. Front. Biosci., Landmark Ed. 15, 25-34.

(20) Nowak, R. G., Bentzen, S. M., Ravel, J., Crowell, T. A., Dauda, W., Ma, B., Liu, H., Blattner, W. A., Baral, S. D., and Charurat, M. E. (2017) Rectal microbiota among HIV-uninfected, untreated HIV, and treated HIV-infected men who have sex with men (MSM) in Nigeria. AIDS 31 (6), 857-862.

(21) Ji, Y., Zhang, F., Zhang, R., Shen, Y., Liu, L., Wang, J., Yang, J., Tang, Q., Xun, J., Qi, T., Wang, Z., Song, W., Tang, Y., Chen, J., and Lu, H. (2018) Changes in intestinal microbiota in HIV-1-infected subjects following cART initiation: influence of CD4+ T cell count. Emerging Microbes Infect. 7 (1), 1-4.

(22) Pinto-Cardoso, S., Lozupone, C., Briceño, O., Alva-Hernández, S., Téllez, N., Adriana, A., Murakami-Ogasawara, A., and Reyes-Terán, G. (2017) Fecal bacterial communities in treated HIV infected individuals on two antiretroviral regimens. Sci. Rep. 7, 43741.

(23) Li, S. X., Armstrong, A. J., Neff, C. P., Shaffer, M., Lozupone, C. A., and Palmer, B. E. (2016) Complexities of gut microbiome dysbiosis in the context of HIV infection and antiretroviral therapy. Clin. Pharmacol. Ther. 99 (6), 600-611.

(24) Noguera-Julian, M., Rocafort, M., Guillén, Y., Rivera, J., Casadellà, M., Nowak, P., Hildebrand, F., Zeller, G., Parera, M., Bellido, R., et al. (2016) Gut microbiota linked to sexual preference and HIV infection. EBioMedicine 5, 135-146.

(25) Lozupone, C. A., Rhodes, M. E., Neff, C. P., Fontenot, A. P., Campbell, T. B., and Palmer, B. E. (2014) HIV-induced alteration in gut microbiota: driving factors, consequences, and effects of antiretroviral therapy. Gut Microbes. 5 (4), 562-570.

(26) Vujkovic-Cvijin, I., Dunham, R. M., Iwai, S., Maher, M. C., Albright, R. G., Broadhurst, M. J., Hernandez, R. D., Lederman, M. M., Huang, Y., Somsouk, M., Deeks, S. G., Hunt, P. W., Lynch, S. V., and McCune, J. M. (2013) Dysbiosis of the gut microbiota is associated with HIV disease progression and tryptophan catabolism. Sci. Transl. Med. 5 (193), 193ra191-193ra191.

(27) Mutlu, E. A., Keshavarzian, A., Losurdo, J., Swanson, G., Siewe, B., Forsyth, C., French, A., DeMarais, P., Sun, Y., Koenig, L., Cox, S., Engen, P., Chakradeo, P., Abbasi, R., Gorenz, A., Burns, C., and Landay, A. (2014) A compositional look at the human gastrointestinal microbiome and immune activation parameters in HIV infected subjects. PLoS Pathog. 10 (2), e1003829.

(28) Vázquez-Castellanos, J., Serrano-Villar, S., Latorre, A., Artacho, A., Ferrus, M., Madrid, N., Vallejo, A., Sainz, T., Martinez-Botas, J., Ferrando-Martinez, S., Vera, M., Dronda, F., Leal, M., Del Romero, J., Moreno, S., Estrada, V., Gosalbes, M. J., and Moya, A. (2015) Altered metabolism of gut microbiota contributes to chronic immune activation in HIV-infected individuals. Mucosal Immunol. 8 (4), 760-722.

(29) Elwell, L. P., Ferone, R., Freeman, G. A., Fyfe, J. A., Hill, J. A., Ray, P. H., Richards, C. A., Singer, S. C., Knick, V. B., and Rideout, J. L. (1987) Antibacterial activity and mechanism of action of $3^{\prime}$-azido-3'- deoxythymidine (BW A509U). Antimicrob. Agents Chemother. 31 (2), 274-280.

(30) Lewin, C., Watt, B., Paton, R., and Amyes, S. (1990) Isolation of zidovudine resistant Escherichia coli from AIDS patients. FEMS Microbiol. Lett. 70 (2), 141-143.

(31) Eckburg, P. B., Bik, E. M., Bernstein, C. N., Purdom, E., Dethlefsen, L., Sargent, M., Gill, S. R., Nelson, K. E., and Relman, D. A. (2005) Diversity of the human intestinal microbial flora. Science 308 (5728), 1635-1638.

(32) Reid, G., Howard, J., and Gan, B. S. (2001) Can bacterial interference prevent infection? Trends Microbiol. 9 (9), 424-428.

(33) Cho, I., and Blaser, M. J. (2012) The human microbiome: at the interface of health and disease. Nat. Rev. Genet. 13 (4), 260-270.

(34) Huttenhower, C., Gevers, D., Knight, R., Abubucker, S., Badger, J. H., Chinwalla, A. T., Creasy, H. H., Earl, A. M., FitzGerald, M. G., and Fulton, R. S. (2012) Structure, function and diversity of the healthy human microbiome. Nature 486 (7402), 207-214.

(35) Gao, Y.-D., Zhao, Y., and Huang, J. (2014) Metabolic modeling of common Escherichia coli strains in human gut microbiome. BioMed Res. Int. 2014, 1.

(36) Vrouenraets, S. M., Wit, F. W., Tongeren, J. v., and Lange, J. M. (2007) Efavirenz: a review. Expert Opin. Pharmacother. 8 (6), 851-871.

(37) Shilaih, M., Angst, D. C., Marzel, A., Bonhoeffer, S., Günthard, H. F., and Kouyos, R. D. (2017) Antibacterial effects of antiretrovirals, potential implications for microbiome studies in HIV. Antiviral Ther. 23 (1), 91-94.

(38) Oswald, S., Meyer zu Schwabedissen, H., Nassif, A., Modess, C., Desta, Z., Ogburn, E., Mostertz, J., Keiser, M., Jia, J., Hubeny, A., Ulrich, A., Runge, D., Marinova, M., Lütjohann, D., Kroemer, H., and Siegmund, W. (2012) Impact of efavirenz on intestinal metabolism and transport: insights from an interaction study with ezetimibe in healthy volunteers. Clin. Pharmacol. Ther. 91 (3), 506-513.

(39) Bengtson, A. M., Pence, B. W., Eaton, E. F., Edwards, J. K., Eron, J. J., Mathews, W. C., Mollan, K., Moore, R. D., O'Cleirigh, C., Geng, E., and Mugavero, M. J. (2018) Patterns of efavirenz use as first-line antiretroviral therapy in the United States: 1999-2015. Antiviral Ther. 23 (4), 363-372.

(40) Kryst, J., Kawalec, P., and Pilc, A. (2015) Efavirenz-based regimens in antiretroviral-naive HIV-infected patients: a systematic review and meta-analysis of randomized controlled trials. PLoS One 10 (5), e0124279.

(41) Department of Health and Human Services (2013) Panel on Antiretroviral Guidelines for Adults and Adolescents Guidelines for the use of antiretroviral agents in HIV-1-infected adults and adolescents. http://aidsinfo.nih.gov/contentfiles/lvguidelines/ AdultandAdolescentGL.pdf (accessed 2019-11-25).

(42) Department of Health and Human Services; Henry J. Kaiser Family Foundation. (2000) Guidelines for the use of antiretroviral agents in HIV-infected adults and adolescents, January 28, 2000 by the Panel on Clinical Practices for Treatment of HIV Infection. HIV clinical trials 1 (1), 60-110.

(43) Hecht, M., Erber, S., Harrer, T., Klinker, H., Roth, T., Parsch, H., Fiebig, N., Fietkau, R., and Distel, L. V. (2015) Efavirenz has the highest anti-proliferative effect of non-nucleoside reverse transcriptase inhibitors against pancreatic cancer cells. PLoS One 10 (6), e0130277.

(44) Machado, M., Sanches-Vaz, M., Cruz, J. P., Mendes, A. M., and Prudêncio, M. (2017) Inhibition of plasmodium hepatic infection by antiretroviral compounds. Front. Cell. Infect. Microbiol. 7, 329.

(45) Costa, S., Machado, M., Cavadas, C., and do Céu Sousa, M. J. P. r. (2016) Antileishmanial activity of antiretroviral drugs combined with miltefosine. Parasitol. Res. 115 (10), 3881-3887.

(46) Turnbaugh, P. J., Ley, R. E., Mahowald, M. A., Magrini, V., Mardis, E. R., and Gordon, J. I. (2006) An obesity-associated gut microbiome with increased capacity for energy harvest. Nature 444 (7122), 1027-1031.

(47) Wexler, H. M. (2007) Bacteroides: the good, the bad, and the nitty-gritty. Clin. Microbiol. Rev. 20 (4), 593-621.

(48) European Medicines Agency (2012) Guideline on the investigation of drug interactions. CPMP/EWP/560/95/Rev. 1 Corr. 2, London, UK. 
(49) Klatt, N. R., Cheu, R., Birse, K., Zevin, A. S., Perner, M., NoëlRomas, L., Grobler, A., Westmacott, G., Xie, I. Y., Butler, J., et al. (2017) Vaginal bacteria modify HIV tenofovir microbicide efficacy in African women. Science 356 (6341), 938-945.

(50) Serrano-Villar, S., Vázquez-Castellanos, J. F., Vallejo, A., Latorre, A., Sainz, T., Ferrando-Martínez, S., Rojo, D., Martínez-Botas, J., del Romero, J., Madrid, N., Leal, M., Mosele, J. I., Motilva, M. J., Barbas, C., Ferrer, M., Moya, A., Moreno, S., Gosalbes, M. J., and Estrada, V. (2017) The effects of prebiotics on microbial dysbiosis, butyrate production and immunity in HIV-infected subjects. Mucosal Immunol. 10 (5), 1279-1293.

(51) Vázquez-Castellanos, J. F., Serrano-Villar, S., JiménezHernández, N., Soto del Rio, M. D., Gayo, S., Rojo, D., Ferrer, M., Barbas, C., Moreno, S., Estrada, V., Rattei, T., Latorre, A., Moya, A., and Gosalbes, M. J. (2018) Interplay between gut microbiota metabolism and inflammation in HIV infection. ISME J. 12 (8), 1964-1976.

(52) Pinto-Cardoso, S., Klatt, N. R., and Reyes-Terán, G. (2018) Impact of antiretroviral drugs on the microbiome: unknown answers to important questions. Curr. Opin. HIV AIDS 13 (1), 53-60.

(53) Bandera, A., De Benedetto, I., Bozzi, G., and Gori, A. (2018) Altered gut microbiome composition in HIV infection: causes, effects and potential intervention. Curr. Opin. HIV AIDS 13 (1), 73-80.

(54) Sortino, O., Phanuphak, N., Schuetz, A., Ortiz, A. M., Chomchey, N., Belkaid, Y., Davis, J., Mystakelis, H. A., Quiñones, M., Deleage, C., Ingram, B., Rerknimitr, R., Pinyakorn, S., Rupert, A., Robb, M. L., Ananworanich, J., Brenchley, J., and Sereti, I. (2020) Impact of Acute HIV Infection and Early Antiretroviral Therapy on the Human Gut Microbiome. Open Forum Infect. Dis. 7 (12), ofz367.

(55) Villanueva-Millán, M. J., Pérez-Matute, P., Recio-Fernández, E., Lezana Rosales, J. M., and Oteo, J. A. (2017) Differential effects of antiretrovirals on microbial translocation and gut microbiota composition of HIV-infected patients. J. Int. AIDS Soc. 20 (1), 21526. (56) Williams, B., Landay, A., and Presti, R. M. (2016) Microbiome alterations in HIV infection a review. Cell. Microbiol. 18 (5), 645-651.

(57) Kim, C. J., Walmsley, S. L., Raboud, J. M., Kovacs, C., Coburn, B., Rousseau, R., Reinhard, R., Rosenes, R., and Kaul, R. (2016) Can probiotics reduce inflammation and enhance gut immune health in people living with HIV: study designs for the Probiotic Visbiome for Inflammation and Translocation (PROOV IT) pilot trials. HIV Clin. Trials 17 (4), 147-157.

(58) Gori, A., Rizzardini, G., Van't Land, B., Amor, K. B., Van Schaik, J., Torti, C., Quirino, T., Tincati, C., Bandera, A., Knol, J., BenlhassanChahour, K., Trabattoni, D., Bray, D., Vriesema, A., Welling, G., Garssen, J., and Clerici, M. (2011) Specific prebiotics modulate gut microbiota and immune activation in HAART-naive HIV-infected adults: results of the "COPA" pilot randomized trial. Mucosal Immunol. 4 (5), 554-563.

(59) Serrano-Villar, S., de Lagarde, M., Vázquez-Castellanos, J., Vallejo, A., Bernadino, J. I., Madrid, N., Matarranz, M., Díaz-Santiago, A., Gutiérrez, C., Cabello, A., Villar-García, J., Blanco, J. R., Bisbal, O., Sainz, T., Moya, A., Moreno, S., Gosalbes, M. J., and Estrada, V. (2018) Effects of immunonutrition in advanced human immunodeficiency virus disease: a randomized placebo-controlled clinical trial (promaltia study). Clin. Infect. Dis. 68 (1), 120-130.

(60) Stiksrud, B., Nowak, P., Nwosu, F. C., Kvale, D., Thalme, A., Sonnerborg, A., Ueland, P. M., Holm, K., Birkeland, S.-E., Dahm, A. E. A., Sandset, P. M., Rudi, K., Hov, J. R., Dyrhol-Riise, A. M., and Trøseid, M. (2015) Reduced levels of D-dimer and changes in gut microbiota composition after probiotic intervention in HIV-infected individuals on stable ART. JAIDS, J. Acquired Immune Defic. Syndr. 70 (4), 329-337. (61) Hensley-McBain, T., Zevin, A. S., Manuzak, J., Smith, E., Gile, J., Miller, C., Agricola, B., Katze, M., Reeves, R. K., Kraft, C. S., Langevin, S., and Klatt, N. R. (2016) Effects of fecal microbial transplantation on microbiome and immunity in simian immunodeficiency virus-infected macaques. J. Virol. 90 (10), 4981-4989.

(62) Vujkovic-Cvijin, I., Rutishauser, R. L., Pao, M., Hunt, P. W., Lynch, S. V., McCune, J. M., and Somsouk, M. (2017) Limited engraftment of donor microbiome via one-time fecal microbial transplantation in treated HIV-infected individuals. Gut Microbes. 8 (5), 440-450.

(63) Cihlar, T., and Fordyce, M. (2016) Current status and prospects of HIV treatment. Curr. Opin. Virol. 18, 50-56.

(64) Department of Health and Human Services (2019) Panel on Antiretroviral Guidelines for Adults and Adolescents. Guidelines for the Use of Antiretroviral Agents in Adults and Adolescents with HIV. http://www.aidsinfo.nih.gov/ContentFiles/AdultandAdolescentGL. pdf (accessed 2019-11-25).

(65) Caporaso, J. G., Kuczynski, J., Stombaugh, J., Bittinger, K., Bushman, F. D., Costello, E. K., Fierer, N., Pena, A. G., Goodrich, J. K., Gordon, J. I., Huttley, G. A., Kelley, S. T., Knights, D., Koenig, J. E., Ley, R. E., Lozupone, C. A., McDonald, D., Muegge, B. D., Pirrung, M., Reeder, J., Sevinsky, J. R., Turnbaugh, P. J., Walters, W. A., Widmann, J., Yatsunenko, T., Zaneveld, J., and Knight, R. (2010) QIIME allows analysis of high-throughput community sequencing data. Nat. Methods 7 (5), 335-336.

(66) Asnicar, F., Weingart, G., Tickle, T. L., Huttenhower, C., and Segata, N. (2015) Compact graphical representation of phylogenetic data and metadata with GraPhlAn. PeerJ 3, e1029.

(67) European Committee on Antimicrobial Susceptibility Testing (2016) Recommendations for MIC determination of colistin (polymyxin E) as recommended by the joint CLSI-EUCAST Polymyxin Breakpoints Working Group. http://www.eucast.org/ fileadmin/src/media/PDFs/EUCAST_files/General_documents/ Recommendations for MIC determination of colistin March . pdf (accessed 2019-11-12).

(68) Rosenblatt, J., Murray, P., Sonnenwirth, A., and Joyce, J. (1979) Comparison of anaerobic susceptibility results obtained by different methods. Antimicrob. Agents Chemother. 15 (3), 351-355.

(69) Jousimies-Somer, H. (2002) Wadsworth-KTL anaerobic bacteriology manual, 6th ed., Star Publ.

(70) Murray, P. R., Baron, E., Pfaller, M., Tenover, F., and Yolken, R. H. (2003) Manual of Clinical Microbiology, ASM Press, Washington, DC.

(71) Garg, R., Kaistha, N., Gupta, V., and Chander, J. (2014) Isolation, identification and antimicrobial susceptibility of anaerobic bacteria: A study re-emphasizing its role. J. Clin. Diagn. Res. 8 (11), DL01-DL02.

(72) Barreau, M., Pagnier, I., and La Scola, B. (2013) Improving the identification of anaerobes in the clinical microbiology laboratory through MALDI-TOF mass spectrometry. Anaerobe 22, 123-125.

(73) Leber, A. L. (2016) Clinical microbiology procedures handbook, Vol. 3, ASM press, Washington, DC.

(74) Clinical and Laboratory Standards Institute (2019) Performance standards for antimicrobial susceptibility testing; 29th informational supplement. CLSI document M100-S29.

(75) Brook, I., Wexler, H. M., and Goldstein, E. J. (2013) Antianaerobic antimicrobials: spectrum and susceptibility testing. Clin. Microbiol. Rev. 26 (3), 526-546.

(76) Schuetz, A. N. (2014) Antimicrobial resistance and susceptibility testing of anaerobic bacteria. Clin. Infect. Dis. 59 (5), 698-705. 